\title{
Féeries
}

Études sur le conte merveilleux, XVII ${ }^{\mathrm{e}} \mathrm{XIX}{ }^{\mathrm{e}}$ siècle

$11 \mid 2014$

L'illustration des contes

\section{Walter Crane lit Barbe bleue : amour, violence... et politique}

Walter Crane reads Blue Beard: love, violence... and politics

\section{François Fièvre}

\section{(2) OpenEdition}

1 Journals

Édition électronique

URL : http://journals.openedition.org/feeries/939

DOI : $10.4000 /$ feeries.939

ISSN : 1957-7753

Éditeur

UGA Éditions/Université Grenoble Alpes

Édition imprimée

Date de publication : 19 décembre 2014

Pagination : 99-123

ISBN : 978-2-84310-281-3

ISSN : 1766-2842

Référence électronique

François Fièvre, « Walter Crane lit Barbe bleue : amour, violence... et politique », Féeries [En ligne], 11 |

2014, mis en ligne le 19 décembre 2015, consulté le 30 octobre 2020. URL : http://

journals.openedition.org/feeries/939; DOI : https://doi.org/10.4000/feeries.939

\section{(c) Féeries}




\section{WALTER CRANE LIT «BARBE BLEUE» : AMOUR, VIOLENCE... ET POLITIQUE}

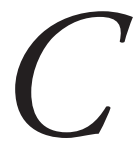

HRISTOPHE MARTIN ÉNONCE LE PARADOXE selon lequel l'illustration paraitrait naturellement liée au conte de fées mais resterait somme toute rare aux débuts du genre, c'est-à-dire au XviII ${ }^{\mathrm{e}}$ siècle $^{\mathrm{I}}$. Ce n'est en effet qu'au siècle suivant que le conte de fées rencontre l'image de manière massive. Cette rencontre "tardive" s'explique d'abord par la multiplication, à l'ère de la révolution industrielle, des technologies de reproduction de l'image imprimée (lithographie, gravure sur bois de bout, gravure sur acier...), qui rendent l'illustration beaucoup moins coûteuse qu'au siècle précédent. Mais surtout elle s'explique par la rencontre du genre littéraire avec le champ éditorial de la littérature pour enfants, alors en plein essor, et qui a pour caractéristique d'être la plupart du temps illustrée.

Une étape importante dans l'histoire de l'illustration pour enfants est l'émergence, au milieu du XIx ${ }^{e}$ siècle, de la forme éditoriale de l'album pour enfants. Et dans ce domaine, Walter Crane est, en Angleterre, un artiste fondamental : c'est avec les toy books («livres jouets») de l'artiste anglais que le conte de fées entre de plain pied dans le champ de l'album pour enfants. Par ailleurs, Walter Crane a la particularité de donner une véritable interprétation artistique des textes qu'il illustre, à travers une composition dense en détails symboliques, qui permettent de rendre en images une partie du sens contenu dans le texte, et non seulement sa lettre. Comment Walter Crane interprète-t-il les contes de fées classiques français, bien connus pour leur richesse sémantique qui a donné lieu à nombre d'exégèses? Par mesure d'économie, et parce que c'est l'un des toy books les plus riches réalisés par l'artiste, nous ne nous intéresserons qu’à un seul conte, Bluebeard («Barbe bleue»), publié par Routledge en I875 dans la

I. C. Martin, "L'illustration du conte de fées (1697-1789)", Cahiers de l'Association internationale des études françaises, $\mathrm{n}^{\circ} 57,2005$, p. I13-132. 
collection «Sixpenny Series". Après avoir évoqué quelques éléments de contextualisation historique, et notamment le problème de la paternité du texte, nous examinerons donc l'interprétation visuelle du conte, avec ses exigences en matière de clarté narrative et ses richesses symboliques, ce qui nous amènera en dernier lieu à l'hypothèse d'une interprétation politique de ce célèbre conte de Perrault.

\section{Contextualisation : genre et paternité des albums de Walter Crane}

\section{Les toy books de Routledge}

Si l'on doit opérer une distinction entre "album» et "livre illustré», il faut adopter un sens étroit du second terme : un livre illustré est alors un livre composé typographiquement selon les règles traditionnelles de la mise en pages, auquel on vient adjoindre des illustrations qui ne viennent que très peu perturber l'écoulement typographique du texte - la mise en pages suit d'abord la logique spatiale (le "design») du compositeur typographique, avant de suivre celle d'un enchaînement d'images ou d'une dynamique iconotextuelle. À l'inverse, l'album est avant tout composé d'images, non dans le sens où celles-ci occuperaient quantitativement plus de place que le texte, mais au sens où c'est leur enchaînement qui vient donner sa place au texte, et non plus l'inverse. Une étape importante de l'histoire de ce genre éditorial se déroule en Angleterre, à Londres, où à partir de 1865 Walter Crane collabore avec Edmund Evans pour produire un certain nombre de livres d'images — appelés indifféremment «picture books» («livres d'images») ou "toy books» — où c'est l'ordre et le rythme des images qui viennent régler l'écoulement typographique du texte ${ }^{3}$.

Walter Crane n'est toutefois pas le premier artiste à donner dans le picture book. Quand il commence à travailler dans ce domaine, il ne fait

2. Deux collections de toy books ont paru chez Routledge : la "Sixpenny Series» (valant, comme son nom l'indique, six pence) où texte et image sont présents sur une même page, et la «Shilling Series» (valant un shilling) où le texte est imprimé à part. Pour une liste quasi complète des toy books de Walter Crane, voir I. Spencer, Walter Crane, New York, Macmillan, I975, p. 202. Nous ne parlerons ici que de l'édition Routledge de Bluebeard, et ne prendrons donc pas en compte dans notre analyse les éléments périphériques (couverture, page de titre, etc.) ajoutés dans les rééditions John Lane de la fin du siècle, par ailleurs très intéressants — pour des raisons techniques toutefois, nos illustrations proviennent d'une réédition John Lane.

3. Voir sur ce point notre article «L'œuvre de Walter Crane, Kate Greenaway et Randolph Caldecott, une piste pour une définition de l'album", Strene [en ligne], $\mathrm{n}^{\circ} 3,2012$, mis en ligne le Is février 20I2, consulté le 25 mars 20I4, <http://strenae.revues.org/583>. 
que s'intégrer à une logique éditoriale préexistante, à l'intérieur de laquelle ont œuvré bien d'autres illustrateurs plus ou moins anonymes avant lui, dont le talentueux et oublié Charles Henry Bennett ${ }^{4}$. La nouveauté que Walter Crane apporte au genre avec son imprimeur Edmund Evans est l'adoption, au fur et à mesure des années, de deux éléments essentiels : une «ligne claire» qui cerne les figures de manière précise et ferme, et réduit le modelé à l'essentiel's, et une mise en pages où le texte est articulé plastiquement aux images, et ne se contente pas d'occuper le bas de la page, comme c'est souvent le cas dans les livres d'images pour enfants de l'époque 6 . Autre élément important, l'impression en couleurs, à propos de laquelle il faut préciser deux choses. D’une part, elle ne date pas de cette époque et est, dans le cadre de l'édition anglaise pour enfants, utilisée au moins depuis les années I840. D’autre part, la nouveauté des livres de Crane, toute relative dans les années I860, bien réelle dans les années I870, est le soin pris à l'impression des images par Edmund Evans, qui autorise un nombre de couleurs important, rare chez d'autres imprimeurs, pouvant aller jusqu'à quatre couleurs en plus du noir voire davantage, l'augmentation de la palette allant de pair avec un nouveau style graphique qui abandonne les hachures ${ }^{7}$.

\section{Un problème de paternité}

Un problème d'auctorialité, familier à ceux qui s'intéressent à l'histoire éditoriale des contes, se pose aussi au sujet des albums édités par Routledge, la plupart du temps sans mention d'auteur. Dans le cas qui nous intéresse, la paternité des images est néanmoins bien reconnaissable par la présence d'une signature qui associe les initiales de l'artiste au dessin, parfois

4. Pour un aperçu du travail de ces différents illustrateurs, voir le recueil conservé à la Bibliothèque nationale de France, Routledge, Aunt Mavor's Toy Books, Londres, Routledge, I868, cote : R 84498460 , où on trouve des toy books de W. Crane, mais aussi de C. Bennett et d'autres illustrateurs anonymes. L'histoire des albums de cette période, édités chez Routledge ou ailleurs, reste à écrire.

5. Ce souci apparaît à partir de Sing a Song of Sixpence, en I866, mais est radicalisé à partir de I870, quand W. Crane commence à importer l'esthétique des xylographies japonaises dans ses picture books, par exemple dans The Fairy Ship (1870).

6. Je présente ces deux éléments comme des esquisses «à première vue» du corpus, et il faudrait bien sûr engager une analyse plus fine des apports de W. Crane dans le contexte des picture books des années I850-1870, qui sont mal connus car peu étudiés. L'apport d'illustrateurs comme C. Bennett ne doit notamment pas être sous-estimé.

7. Voir S. Hyde, "The production of Walter Crane's children's books", dans G. Smith et S. Hyde (éd.), Walter Crane, I845-19I5. Artist, Designer and Socialist, cat. expo. (Manchester, Whitworth Art Gallery), Londres, Lund Humphries, 1989, p. 26-29. 
schématique, d'une grue homonyme, ainsi que par une couverture générique - exceptionnelle pour ce type de production, rappelant le succès dont pouvait se prévaloir l'artiste dans les années I870 - qui rassemble à partir de I873 différents titres sous une même étiquette, Walter Crane's Toy Books ("Les livres jouets de Walter Crane»). Mais qu'en estil, par ailleurs, de la paternité du texte? Personne n'a vraiment cherché à résoudre ce problème d'attribution, la prééminence de l'image suffisant la plupart du temps pour attribuer la paternité du tout, images et texte compris, à l'artiste ${ }^{8}$. Isobel Spencer nous informe que les abécédaires et certaines histoires auraient été écrits par Walter Crane et sa sœur Lucy, mais sans davantage de précisions, ni référence qui nous permette d'approfondir la question'. Un document nous donne un indice : il s'agit de l'introduction à l'ouvrage The Art and Formation of Taste ("L'Art et la Formation du goût»), recueil de textes critiques de Lucy Crane sur les beaux-arts et les arts décoratifs, qui précise :

Elle écrivit également les vers originaux, et les versions rimées de légendes enfantines bien connues pour les toy books en couleur de son frère (Walter Crane). Parmi les vers originaux on peut citer "How Jessie was Lost" ["Comment Jessie sest perdue»], "The Adventures of Puffy" ["Les Aventures de Puffy"], "Annie and Jack in London" ["Annie et Jack à Londres"], qui parurent séparément dans cette collection ${ }^{\mathrm{I}}$.

Sans doute doit-on donc considérer comme à peu près certain que c'est Lucy Crane qui fut responsable de l'adaptation en vers de ces «well-known nursery legends» («légendes enfantines bien connues») que sont Little Red Riding Hood («Le Petit Chaperon rouge»), Bluebeard, etc. Toutefois, un détail reste à aborder.

En effet, quand on regarde de près le texte de l'album de Walter Crane pour Jack in the Beanstalk ("Jack et le Haricot magique», I875), on se rend compte, comme l'a bien montré Morna O’Neill, que le récit traditionnel a été considérablement adapté pour faire passer un message socialiste, et ce sur le modèle de l'adaptation qu'avait déjà faite du conte

8. Ainsi de J. Hichberger ("Walter Crane’s children’s books and mid-Victorian attitudes to childhood ", dans Smith et Hyde [éd.], ibid., p. 35) à propos de The Frog Prince ("Le Prince Grenouille», tiré de Grimm) : "the text, probably by Crane» ("le texte, probablement de Crane»).

9. I. Spencer, ouvr. cité, p. 46.

I0. "She also wrote the original verses, and rhymed versions of well-known nursery legends for her brother's (Walter Crane) coloured toy-books. Among the original verses may be named "How Jessie was Lost", "The Adventures of Puffy", "Annie and Jack in London" which severally appeared in the series." ("In Memory of Lucy Crane», dans L. Crane, Art and the Formation of Taste, Londres, Macmillan, I882, p. XI.) Le texte est rédigé au passé dans la mesure où, à la date de publication de l'ouvrage, L. Crane était décédée. 
William James Linton, maître de l'artiste, dans un livre pour enfants paru en $1868^{\text {II. }}$ Si Lucy Crane a pu être proche des idées de John Ruskin et de William Morris, rien n'indique qu'elle ait été, à l'instar de son frère, une socialiste engagée; surtout, c'est Walter Crane et non sa sœur Lucy qui fut proche de Linton : la proximité avec la version de Linton comme le poids idéologique de l'adaptation de ce conte témoignent en faveur d'une intervention de l'artiste sur le texte. Dès lors, pourquoi ne pas admettre aussi cette possibilité pour les autres adaptations? Le fait est que Walter et Lucy ont très souvent travaillé ensemble, et qu'il est difficile sans autre document d'archives d'opérer une distinction stricte entre les interventions de l'un et de l'autre au cours du travail sur les textes. Au final, on peut sans doute avancer ce partage des tâches : la lettre du texte semble devoir tomber sous la responsabilité de Lucy, alors que la sélection des histoires, et de temps à autre le contenu de tel ou tel motif narratif, a pu être l'objet, pour reprendre le mot de Jean-Pierre Collinet à propos des contes de Perrault, d'une "souple collaboration" entre frère et sœur; enfin, les images relèvent entièrement, bien sûr, de la responsabilité de Walter. Mais comme dans le cas des frères Grimm, ou même celui de Charles Perrault et de son fils Pierre Darmancour ${ }^{12}$, il est remarquable que la création des contes ait donné lieu à une telle collaboration familiale. La dimension de «fraternité des arts» vient ici s'ajouter au paradigme du conte «fait en famille», dans la mesure où la peinture vient collaborer avec la poésie ${ }^{\mathrm{I} 3}$.

En dernier lieu, quel texte les Crane ont-ils utilisé comme matériau de départ de Bluebeard: l'original français ou bien une traduction? Lucy Crane maîtrisait la langue française, comme en témoigne l'inclusion de comptines françaises dans The Baby's Bouquet en I878, mais peut-être pas à un niveau suffisant pour avoir aisément accès à la version de Perrault ${ }^{14}$.

II. M. O'Neill, “Art and Labour's Cause is One." Walter Crane and Manchester, I880-I9I5, cat. expo. (Manchester, Whitworth Art Gallery), Manchester, University of Manchester, 2008, p. 25, et note 27 p. 38. Ladaptation de ce conte traditionnel anglais par W. J. Linton prend place dans un recueil intitule The Flower and the Star and Other Stories for Children (Boston, Tickner and Fields, I868) : l'ogre, comme dans l'album de Crane, y vole les richesses de la famille de Jack, à l'instar du capital qui ponctionne les fruits du travail du prolétariat, et Jack ne fait donc que reprendre son bien quand il vole l'ogre.

I2. Voir J.-P. Collinet, «Préface», dans Perrault, Contes, Paris, Gallimard, I98I, p. 25-30, pour le problème d'attribution.

13. En 1882, cette fraternité des arts du lisible et du visible sera mise en avant dans la page de titre pour Household Stories, recueil de contes traduits par Lucy du recueil des frères Grimm et illustré par Walter. Voir F. Fièvre, Le Conte et l'Image. L'illustration des contes de Grimm en Angleterre au XIX ${ }^{e}$ siècle, Tours, Presses universitaires François Rabelais, 20I3, p. 229 et suiv.

I4. L. Crane, pour The Baby's Opera et The Baby's Bouquet, était responsable de la sélection et de l'arrangement musical des chansons, ce qui suppose une affinité minimale avec la langue dans 
Plus probable est l'utilisation d'un texte-source en langue anglaise, en l'occurrence pour Bluebeard les vingt-quatre contes de fées traduits du français par James Robinson Planché, parus chez George Routledge en I858 ${ }^{15}$. Casie E. Hermansson nous informe que, malgré des modifications conséquentes, c'était probablement à l'époque l'édition la plus fiable de contes de fées français ${ }^{16}$, et le fait qu'elle fasse partie du catalogue de Routledge rend probable l'hypothèse selon laquelle l'éditeur a fourni ce matériau à l'illustrateur quand il s'est agi d'adapter des contes au début des années I870. L'incipit de l'album ("Once on a time there lived a man" — "Il était une fois vivait un homme») ressemble beaucoup, notamment du fait du remplacement du traditionnel «upon» par «on» dans la formule consacrée d'introduction, à celui utilisé par Planché ("Once on a time there was a man» — «Il était une fois il y avait un homme»). Autre détail confortant la thèse d'une utilisation de la traduction de Planché, la traduction difficile du discours de la sœur Anne qui, en haut de la tour, dit ne voir «rien que le Soleil qui poudroie, et l'herbe qui verdoie ${ }^{17}$ », qui est traduit par Lucy Crane par "But shining sun and growing grass, and dust upon the road» ("Sinon le soleil qui brille et l'herbe verte qui pousse, et la poussière sur la route») et semble suivre, avec l'image de l'herbe qui pousse et la traduction de "poudre" par "dust», la traduction de Planché «but the sun making dust, and the grass growing green" ("sinon le soleil faisant de la poussière, et l'herbe verte qui pousse»). Toutefois le texte, que ce soit celui de Planché — assez fidèle à l'original —, ou celui de Perrault, est tellement altéré après sa mise en vers qu'il est délicat de trancher en faveur d'une adaptation du texte anglais plutôt que d'une traduction libre de l'original français.

laquelle elles sont écrites. The Baby's Opera ne contient que des comptines en anglais, alors que The Baby's Bouquet contient aussi des comptines en français et en allemand. L. Crane maîtrisait suffisamment l'allemand pour traduire les contes de Grimm (I882) et, d'après le Dictionary of National Biography, maîtrisait semble-t-il aussi l'italien.

15. Four and Twenty Fairy-Tales, Selected from Those of Perrault, and Other Popular Authors, trad. ang. J. R. Planché, Londres, Routledge, I858. Les citations de la traduction de Planché sont faites d'après cet ouvrage, p. 3-7.

I6. C. E. Hermansson, Bluebeard. A Reader's Guide to the English Tradition, Jackson, University Press of Mississippi, 2009, p. III-II2.

I7. Toutes les citations en français du conte sont faites d'après l'édition de J.-P. Collinet (ouvr. cité, p. I47-I54). 


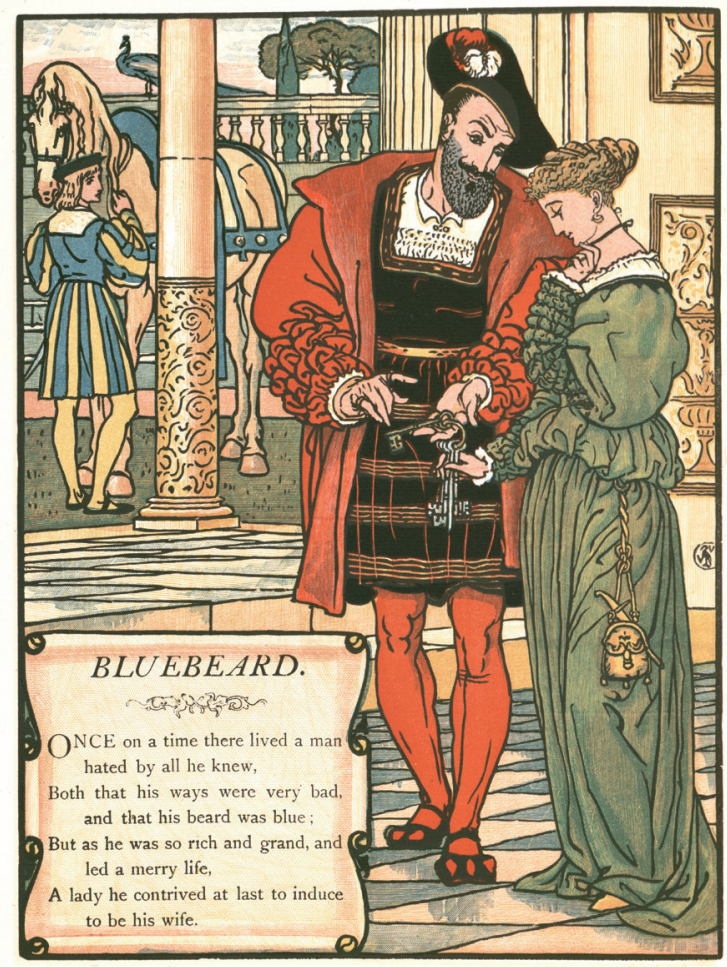

Fig. I. - Walter Crane, chromoxylographie d'Edmund Evans, planche I (p. I) de Bluebeard, Londres, John Lane, 1898.

Université François-Rabelais, Tours.

\section{Une illustration narrative}

Pour commencer l'analyse, examinons la manière dont les personnages sont représentés. Un premier détail, dès la première planche (fig. I), est la barbe de Barbe bleue, d'un bleu très léger, qui témoigne probablement d'une volonté de l'illustrateur de s'inscrire dans la tradition d'interprétation réaliste faisant de ce bleu la nuance, le reflet d'une barbe noire: les traits de la barbe sont imprimés en noir, et c'est sur cette base qu'une ombre bleue a été ajoutée. Par ailleurs, Walter Crane rompt avec la tra- 
dition qui faisait de Barbe bleue un Turc ${ }^{18}$, et ne le représente donc pas affublé d'un turban, d'un cimeterre et d'habits orientaux, mais d'habits tout droits venus de la Renaissance. L'adoption de costumes du XVI ${ }^{\mathrm{e}}$ siècle connote une période qui, même à l'époque de Perrault, relevait de l'«ancien temps» dans lequel prenaient place les récits ${ }^{19}$. Par ailleurs Walter Crane, à la suite des Préraphaélites et dans la mouvance de l'Aesthetic Movement, s'intéresse beaucoup à l'art de la Renaissance, duquel il s'inspire à de nombreuses reprises ${ }^{20}$. Autre possibilité, l'illustrateur pourrait avoir eu en tête l'interprétation du conte par Gustave Doré, qui date de I862, et situait également les contes dans un contexte tiré de l'époque de Rabelais. Enfin, on pourra peut-être reconnaître, dans la forme du visage, la barbe massive mais taillée, certains détails de costume, et surtout la carrure gigantesque des épaules, les modèles du portrait de François I ${ }^{\text {er }}$ par Jean Clouet (Paris, musée du Louvre, $c$. I530), de celui de Jean de Dinteville dans le célèbre tableau Les Ambassadeurs de Hans Holbein le Jeune (Londres, National Gallery, I533), ou bien, encore plus probablement, de celui d'Henry VIII, toujours par Hans Holbein, qui a disparu mais dont il existe de nombreuses copies. Cette dernière hypothèse est très probable, la résonance symbolique du roi aux six femmes, dont l'une (Anne Boleyn) est exécutée, étant parfaite avec le personnage de Barbe bleue, et certains détails comme le chapeau ou les chaussures semblant un emprunt direct.

La femme de Barbe bleue est elle-même habillée d'une robe verte de style Renaissance, qui changera de couleur dans la suite des images. Pour quelle raison, alors que le "code couleur" des personnages semble par ailleurs tout à fait stable d'une image à l'autre ${ }^{21}$ ? Le fait est qu'un laps de temps important se passe à l'intérieur du récit entre le moment où Barbe bleue remet les clefs à son épouse et celui où cette dernière outrepasse

I8. Voir sur ce point C. E. Hermansson, ouvr. cité, p. 5I-66.

19. Comme en témoigne la seconde moralité du conte, qui pour rassurer le lecteur énonce qu' «On voit bien que cette histoire / Est un conte du temps passé». Cette moralité est la seule traduite par Planché.

20. Et notamment de Dürer et d'Holbein, voir F. Fièvre, Le Conte et l'Image..., ouvr. cité, p. 253-256.

2I. On remarquera par ailleurs que la séquentialité des images est garantie par la présence quasi constante du personnage de la femme de Barbe bleue, qui forme véritablement le fil conducteur de la série. La seule image où elle est absente se trouve en planche 5 (fig. 5), où seule la sœur Anne est représentée. Encore celle-ci avait-elle été introduite à l'arrière-plan de la planche précédente, et sera-t-elle encore présente à celui de la suivante : une continuité narrative est ainsi ménagée entre les images à travers une coprésence des personnages. De même les deux frères sont introduits à l'arrière-plan de la planche 6 (fig. 6) avant d'apparaître au premier plan de la planche 7 (fig. 7 ) : W. Crane a visiblement pris le soin de ne pas faire surgir les personnages secondaires au premier plan de l'action sans les avoir présentés au préalable au spectateur, en les faisant apparaître en quelque sorte «en toile de fond» de l'image précédente. 
l'interdiction : le changement de couleur, alors que la coupe du vêtement reste rigoureusement la même, est une manière de signifier ce passage du temps. Le décalage entre le costume de Barbe bleue dans la planche I (fig. I) et les planches suivantes s'explique de la même manière : un certain temps a passé, Barbe bleue a changé de vêtement, et la raison pour laquelle les différents personnages n'en changent pas à partir de la planche 2 est que toute l'action du reste du récit est resserrée au cours d'une même journée, contrairement à ce qui se passait dans l'original de Perrault qui mettait en place un temps un peu plus long. À l'inverse de sa femme, les vêtements de Barbe bleue changent de coupe, mais pas de couleur : il reste vêtu de rouge, couleur symbolique s'il en est pour caractériser un tempérament violent et passionnel. Le fait qu'il passe d'un vêtement noir et rouge en planche I à un autre entièrement rouge dans les planches suivantes marque de ce point de vue la révélation au grand jour de l'aspect sanglant du personnage, qui n'était que latent au début de l'histoire, et est révélé à partir du moment où la porte du cabinet est ouverte. Autre détail de costume : le mince collier de la femme de Barbe bleue, sorte de bandeau noir auquel est attaché un bijou en forme de cœur (fig. 2), semble comme une préfiguration de la décapitation ou de l'égorgement qui la menacent. Ce collier disparaît à partir du moment où la porte du cabinet interdit est ouverte : la menace n'est plus latente mais manifeste, la vérité sur Barbe bleue s'est faite jour, aussi le collier-bandeau comme signe avant-coureur de la violence maritale perd sa raison d'être. Enfin, la chevelure de la belle épousée, au départ relevée en un strict et élaboré chignon, se défait sous le coup de la violence des sentiments et de la brutalité de l'action, d'abord de manière partielle en double page centrale où a lieu l'altercation avec Barbe bleue (fig. 4), puis complètement en planche 6 où l'épouse est poussée dans ses derniers retranchements (fig. 6). 


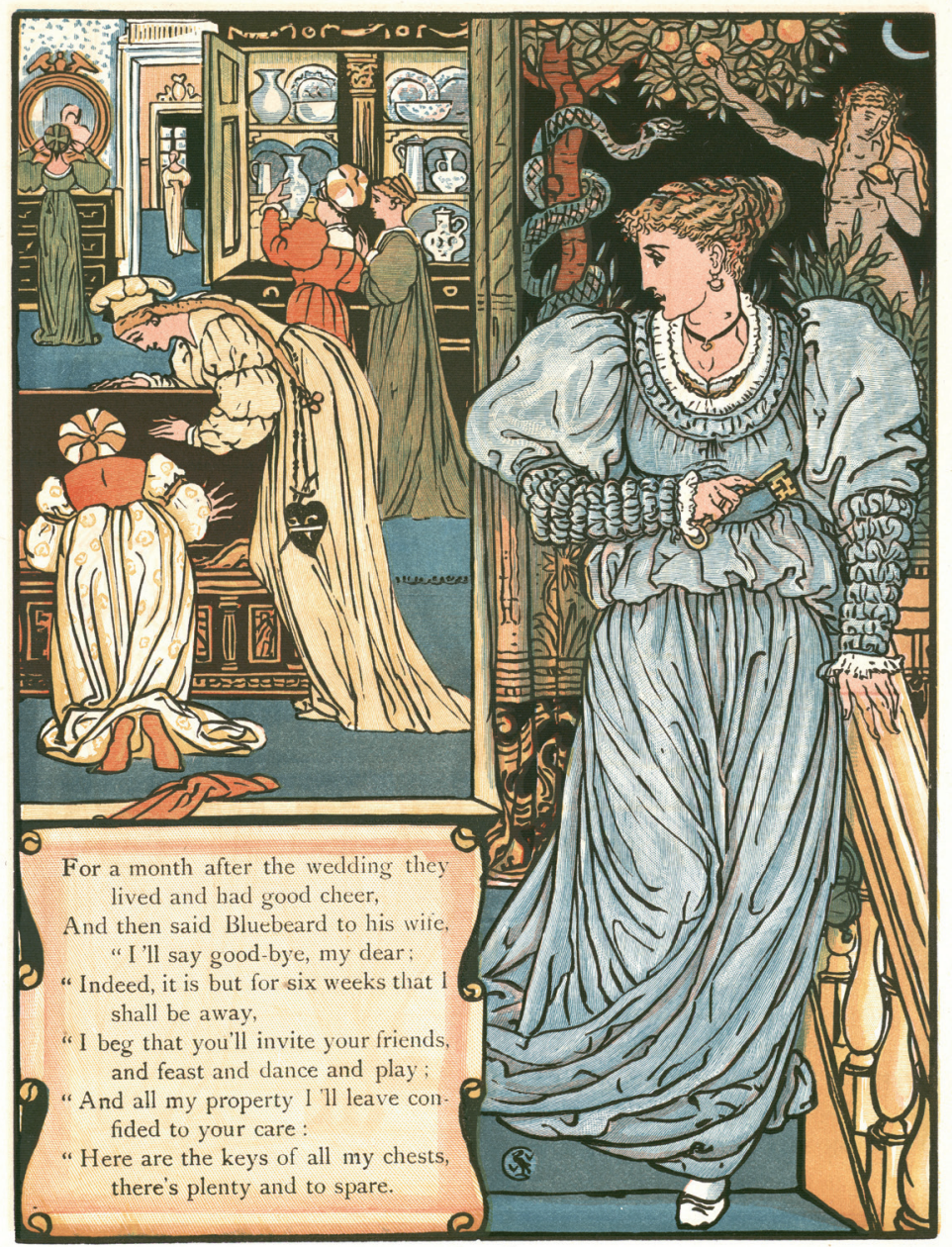

Fig. 2. - Walter Crane, chromoxylographie d'Edmund Evans, planche 2 (p. 2) de Bluebeard, Londres, John Lane, 1898.

Université François-Rabelais, Tours. 


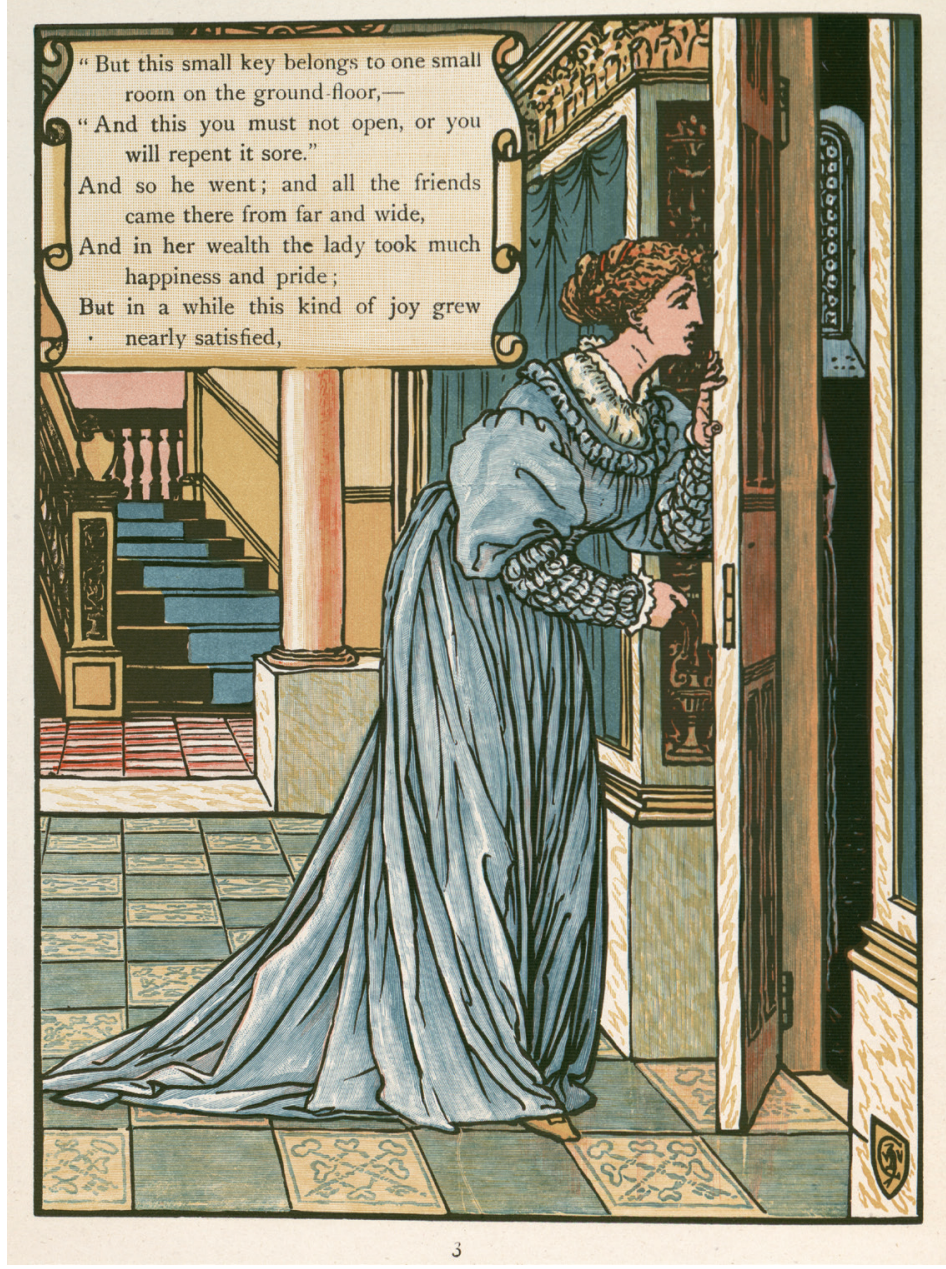

Fig. 3. - Walter Crane, chromoxylographie d'Edmund Evans, planche 3 (p. 3) de Bluebeard, Londres, John Lane, 1898.

Université François-Rabelais, Tours.

D'autres éléments, notamment dans l'organisation de l'espace, permettent de mettre en place de manière claire les éléments du récit et d'organiser les images en séquence narrative, de manière à ce que l'histoire soit parfaitement compréhensible pour un œil enfantin. Par exemple, toujours en planche I (fig. I), le fait de représenter un palefrenier tenant prête une monture à l'arrière-plan permet non seulement de montrer la richesse de Barbe bleue, mais aussi d'indiquer que c'est parce qu'il part en voyage 
qu'il laisse les clefs à sa femme. En planche 3 (fig. 3), l'escalier à l'arrièreplan rappelle celui par lequel est descendue la femme en planche précédente, et permet de rendre cohérent l'espace d'un point de vue narratif autant qu'architectural. Le fait de représenter un espace vertigineux en planche 5 (fig. 5) par l'intermédiaire d'un ensemble de procédés visuels — ligne d'horizon placée en dessous de l'axe horizontal médian, « rupture de format» rendant invisible le bas de la tour, et surtout représentation des toits et du vol d'une hirondelle en dessous de la figure humaine permet de marquer le fait que sœur Anne se trouve véritablement dans les hauteurs du château, hauteurs vers lesquelles elle s'acheminait déjà en planche 4 (fig. 4). En planche 6 (fig. 6), l'illustrateur montre son ambition séquentielle en représentant trois scènes différentes en une seule image: au premier plan, l'épouse de Barbe bleue dans l'escalier menant à la tour, avec en bas Barbe bleue levant son épée et lui ordonnant de descendre; à l'arrière-plan à gauche, en haut des escaliers, la sœur Anne faisant signe aux deux frères de se hâter; à l'arrière-plan à droite, derrière une fenêtre ouverte dans la cage d'escalier, les deux cavaliers galopant épée dégainée vers le château.

Cette tripartition de l'espace permet de rendre compte de deux choses ${ }^{22}$. D'une part, d'une logique narrative voulant que toutes ces actions, dans le texte, se déroulent dans un laps de temps très court : la représentation de la femme de Barbe bleue au premier plan, dans l'espace intermédiaire de l'escalier, situé symboliquement entre délivrance et trépas, permet de faire monter la tension du récit à son comble. D'autant plus que le lecteur voit la libération arriver par la fenêtre, qu'il l'appelle de ses vœux par la figure-relais de la sœur Anne, et qu'il se voit menacé lui-même par Barbe bleue représenté de face - soit faisant face au spectateur, et levant son épée vers lui. Aucun ordre de lecture des différentes scènes n'est visuellement suggéré, si bien que l'on a clairement l'impression que tout se passe en même temps, et donc que «le temps presse». Et d'autre part, d'une logique spatiale rendant compte de l'architecture même du château, nous rappelant que le rez-de-chaussée est le lieu du cabinet interdit symboliquement attaché au mari, et que les appartements de l'étage sont au contraire dévolus à la femme - et même aux femmes dans la mesure où

22. Cette tripartition est visiblement héritée des toutes premières illustrations du conte (manuscrit de 1695 et édition Barbin de 1697), mais est savamment réorchestrée. Il n’y a aucune chance pour que W. Crane ait connu le manuscrit, ni même la toute première édition, mais les gravures de Clouzier ont fait l'objet au XVIII ${ }^{\mathrm{e}}$ siècle d'un certain nombre de réemplois et de copies dont l'artiste a tout à fait pu avoir connaissance. 
en planche 2 ce sont les appartements hauts qui sont investis par les amies de l'épousée ${ }^{23}$. Une organisation symbolique de l'espace fictif du château, donc, qui tend à clarifier le récit en images, et où la construction géométrique de la perspective est appuyée de manière certes à s'inscrire encore une fois dans une filiation avec la peinture de la Renaissance, mais aussi à rendre l'espace du récit parfaitement compréhensible et cohérent.

\section{Une illustration symbolique}

Les images sont par ailleurs jalonnées de motifs symboliques dont la lecture s'avère moins évidente, mais qui permettent d'organiser pour le lecteur cultivé comme un jeu visuel de devinettes et de renvois symboliques. Ainsi de la devise "Gardez le clef» qui permet de marquer l'origine française - et prétendument archaïque, avec ce masculin inventé pour le substantif "clef» — du récit, qui apparaît régulièrement auprès des armoiries de Barbe bleue à partir de la planche 4 - soit à partir du moment où sa véritable nature est révélée. Et des clefs, véritables emblèmes du conte, qui apparaissent en tant que motifs décoratifs dans le carrelage de la planche 3, ou dans le bandeau ornemental supérieur de la chambre de la planche 4. Ou encore des armes de Barbe bleue, qui apparaissent sur le coffre à l'arrière-plan de la planche 4 , puis au premier plan dans les deux planches suivantes : on peut y voir une clef, bien sûr, mais aussi une épée encadrée de deux gouttes de sang, et une tête de mort (fig. 4) ou une figure de pendu (fig. 6), ce qui en dit long sur l'identité symbolique du mari. La silhouette diabolique attachée aux armoiries en planche 5 (fig. 5), dont on retrouve un écho dans la girouette de la tour de droite, dit bien aussi le caractère maléfique du seigneur vêtu de rouge. Le plus intéressant est cependant que Walter Crane, en donnant des armes à Barbe bleue, non seulement se serve du langage de l'héraldique où l'image fait sens par ellemême sans l'aide d'un texte attenant, mais aussi transforme ce terrifiant

23. Nous rappelons que le cabinet interdit se trouve en effet dans le texte de Perrault «au bout de la grande galerie de l'appartement bas»; J.-P. Collinet rapporte à ce propos la description faite par Furetière dans son Dictionnaire : "Le bel appartement, le premier appartement, est celui du premier étage, et est d'ordinaire l'appartement de Madame. L'appartement du bas est celui de Monsieur.» (Cité note 6, p. I50.) On a donc une claire délimitation entre les étages hauts dévolus aux femmes et les étages bas dévolus aux hommes, ce que représente bien Crane dans la double page centrale, où les appartements personnels de la femme (avec son métier à broder et son livre ouvert) se situent dans les hauteurs du château. 
personnage en noble, alors que la version donnée par Perrault en faisait un simple marchand. On y reviendra.

On notera en attendant quatre motifs symboliques importants. Le premier est évidemment la lourde tapisserie de la planche 2, qui représente le péché originel d'Ève au jardin d'Éden, et inscrit ainsi le récit avec son motif de belle curieuse dans son intertextualité biblique. On notera du point de vue de l'organisation de l'espace que l'illustrateur prend bien soin de placer la femme de Barbe bleue dans le même espace bidimensionnel que celui de la tapisserie, inscrivant bien son geste de descente des escaliers dans la lignée symbolique de la Chute. La composition est ainsi clairement découpée par l'axe vertical médian, avec d'un côté le "jardin des plaisirs» avec coffres, belle vaisselle, etc., et de l'autre le mouvement de descente vers l'abîme de la transgression. Le motif de la tapisserie est redoublé par celui de la broderie en double page centrale (fig. 4), l'image suggérant ainsi l'idée que c'est la femme qui est l'autrice de la tapisserie, et qu’elle a ainsi symboliquement déjà tissé son propre destin ${ }^{24}$.

Le deuxième motif est celui du coffre, coffre au trésor en planche 2, mais aussi coffre de mariage en planche 4 , inspiré des cassoni ("coffres») de la Renaissance italienne. L'intertextualité est ici celle du mythe de Pandore, une autre "belle curieuse" qui ouvre la boîte d'où sortent les malheurs du monde. Cet intertexte, qui n'était que suggéré dans le texte de Perrault où Barbe bleue fait mention de ses "coffres-forts, où est [son] or et [son] argent", et de ses "cassettes où sont [ses] pierreries", est clairement mis en avant dans l'illustration de Doré en I862, et repris par l'illustrateur anglais une douzaine d'années plus tard. En planche 4, le coffre se trouve dans les appartements dévolus à l'épouse, et est donc dévolu à son usage, mais en même temps il est décoré des armoiries du mari; il réunit ainsi les deux personnages dans une même symbolique d'union conjugale, qui s'inscrit sous la devise "Gardez le clef» alors même que la serrure, bien voyante, invite à la tentation ${ }^{25}$. Un mariage ambivalent, donc, qui montre et dit de

24. On pourra nous dire à raison que broder n'est pas tisser, et que le lien entre tapisserie de la planche 2 et métier à broder de la planche 4 n'est que conjectural. Il reste que, sans suggérer l'idée que c'est la femme de Barbe bleue qui a réalisé la tapisserie de la planche 2, cette série d'illustrations inscrit indubitablement les activités du tissage et de la broderie, de manière d'ailleurs tout à fait traditionnelle, dans un espace clairement et uniformément féminin : si ce n'est pas la femme de Barbe bleue en tant que telle qui a tissé/brodé son propre destin, c'est du moins la femme "en général» qui s'inscrit d'elle-même, par un lien entre iconographie (Ève) et support (tapisserie/broderie), sous le signe de la curiosité mal placée.

25. Rappelons d'ailleurs que selon certaines traditions la boîte de Pandore (à l'origine une jarre) est un cadeau de mariage donné par les dieux à Pandore et Épiméthée: W. Crane nourrit ici le conte de fées de culture classique. 
ne pas aller voir, un mariage "de la tentation». Mais de quelle tentation s'agit-il?

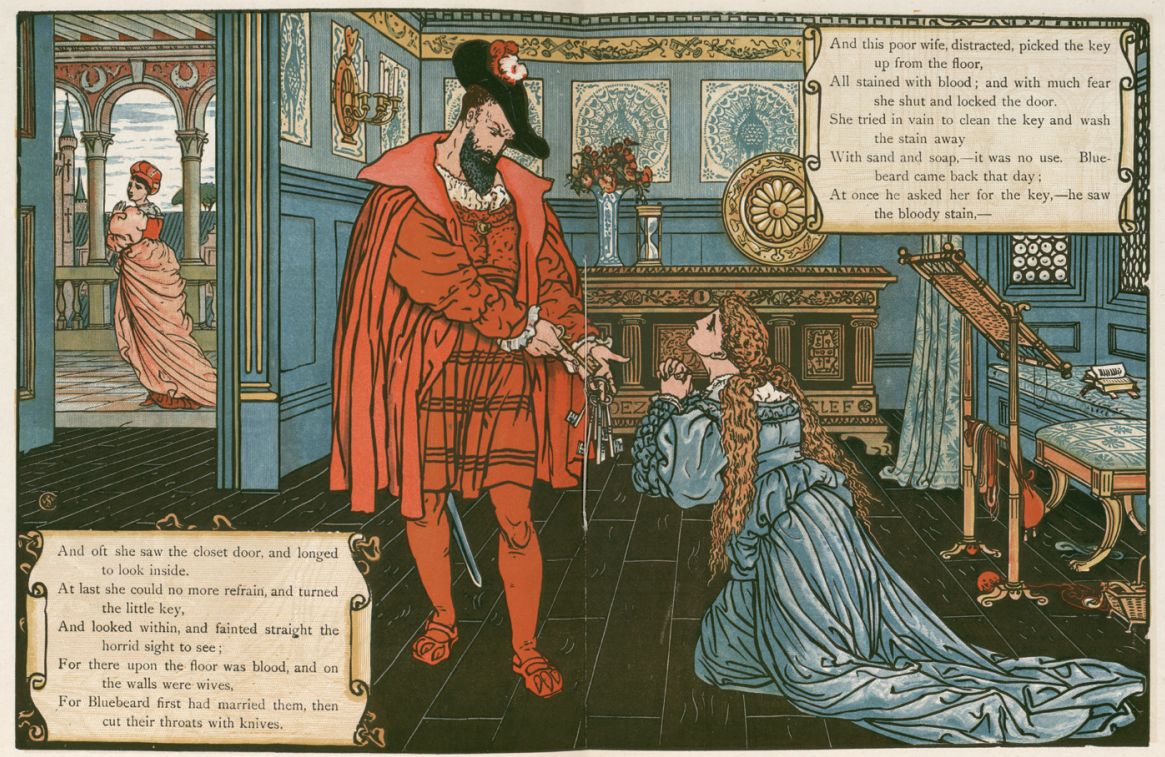

Fig. 4. - Walter Crane, chromoxylographie d'Edmund Evans, planche 4 (p. 4-5) de Bluebeard, Londres, John Lane, 1898.

Université François-Rabelais, Tours.

Un troisième motif est celui du paon, qui en planche I pourrait donner l'impression de n'avoir été placé que pour "meubler" la vue sur le parc, mais qui, repris dans la frise décorative de la chambre en planche 4, met la puce à l'oreille : l'illustrateur n'aurait jamais insisté ainsi sur un motif sans que celui-ci n'ait du sens. En l'occurrence, on peut rappeler que le paon est non seulement un motif récurrent et quasi emblématique, de Whistler à Beardsley, de l'Aesthetic Movement, mais aussi, dans la mythologie grécoromaine, l'animal attaché à la déesse Héra, déesse du mariage - et partant, de la jalousie. La symbolique conjugale du cassone est donc redoublée ici par la figuration ornementale de paons. Rappelons par ailleurs que le paon doit la particularité de ses plumes au transfert sur le plumage de l'oiseau des yeux d'Argos Panoptès, gardien aux cent yeux, mandaté par Héra pour surveiller les infidélités de Zeus, et tué par Hermès. Le paon rappelle donc non seulement l'idée du mariage, mais aussi celle de la vision, et pas de n'importe laquelle : une vision qui jalouse et surveille. Allusion au regard de la femme de Barbe bleue qui a vu l'intérieur du cabinet secret, 
ou bien à celui de Barbe bleue lui-même, qui surveille sa femme d'un œeil terrible? Nous penchons pour la deuxième hypothèse mais, comme tout symbole, le paon est ici fortement ambivalent.

Le quatrième motif est celui du miroir, qu'on aperçoit à l'arrière-plan en planche 2 et qui n'est, à l'instar de la tapisserie, pas mentionné dans le texte de Lucy Crane. Il l'est en revanche dans l'original de Perrault - et également dans la traduction de Planché ${ }^{26}$ :

Elles montèrent ensuite aux garde-meubles, où elles ne pouvaient assez admirer le nombre et la beauté des tapisseries, des lits, des sofas, des cabinets, des guéridons, des tables et des miroirs, où l'on se voyait depuis les pieds jusqu'à la tête, et dont les bordures, les unes de glace, les autres d'argent et de vermeil doré, étaient les plus belles et les plus magnifiques qu'on eût jamais vues.

Significativement, dans le texte de Perrault, le meuble le plus longuement décrit de cette séquence est le miroir, qui est certes admiré pour luimême, mais où surtout l'on peut se voir "depuis les pieds jusqu'à la tête". Espace de contemplation narcissique, le miroir renvoie la belle curieuse à elle-même, et multiplie de ses reflets la jouissance de posséder autant de robes, bijoux et richesses. Une telle mise en abyme de la contemplation narcissique de la possession matérielle est magistralement mise en scène par Walter Crane dans son illustration. Le "paradis terrestre» du gardemeubles où se situent tous les trésors du château est en effet, en planche 2, encadré visuellement en bas par une marche et le bord supérieur du cartel, et à droite par l'épaisseur du mur redoublée de la ligne verticale du bord gauche de la tapisserie. Un tel surencadrement de la scène donne clairement l'impression d'un espace serti dans un autre espace, d'une boîte à l'intérieur de la fenêtre de l'espace visuel du récit, et à l'intérieur de laquelle, dans une mise en abyme vertigineuse, se découpent encore d'autres espaces : celui d'un coffre empli de linge, d'un buffet empli de vaisselle, d'une commode au-dessus de laquelle se trouve un miroir, puis encore d'autres pièces, en enfilade, qui font s'emboîter les espaces les uns dans les autres. La femme nous tournant le dos, agenouillée devant le coffre, est redoublée par celle qui se contemple dans le miroir à l'arrière-plan; les deux invitent le spectateur à s'identifier aux amies de la mariée dans leur parcours spatial et visuel au sein de la "boîte à trésors" vertigineuse et

26. Ce ne sont pas les seuls détails du texte d'origine qu'on retrouve dans l'image et non le texte de l'album : ainsi par exemple du troupeau de moutons en planche 5. Crane n'illustre donc pas tant le récit en vers attenant que le récit d'origine; on a ici affaire à une double interprétation du texte de Perrault, en images et en texte, et non à une série d'images illustrant un texte adapté d'un autre texte. 
infinie du garde-meubles. Pourtant, la femme de Barbe bleue n'est pas satisfaite, et se détourne de cette boîte narcissique et vertigineuse. Comment doit-on interpréter ce mouvement de détournement? De quelle nature est la tentation? Il nous semble que la bonne interprétation ici, avant d'être sexuelle, est politique.

\section{Amour ou politique? Le coffre à plaisirs du capital}

On a eu beau jeu, notamment après Bruno Bettelheim, d'associer le motif de la clef ensanglantée à celui d'un pénis déflorateur, et la découverte du cabinet secret à celle de la sexualité ${ }^{27}$. Nous ne nous prononcerons pas ici sur la pertinence ou non de l'application de telles grilles de lecture psychanalytiques sur le texte de Perrault. Mais nous voulons en revanche nous défendre de la tentation d'une application automatique de telles grilles aux images. L'image n'est pas le texte, et s'il y a une signification psychanalytique à trouver dans «l'inconscient du texte», pour reprendre l'expression de Jean Bellemin-Noël, rien ne dit a priori que cette matière symbolique est véhiculée dans ses images par l'illustrateur. Tout dépend du parti pris par l'artiste : celui-ci pourra certes interpréter le texte dans une direction qui mette au jour cette matière, mais il pourra aussi l'exploiter selon des problématiques qui diffèrent passablement de celles présentes dans le texte d'origine. Que l'image puisse être dotée d'un inconscient au même titre que le texte, nous voulons bien le croire, mais rien n'indique que, dans le cas de l'illustration, l'inconscient iconique se fasse nécessairement l'écho de l'inconscient textuel d'origine. En bref, l'illustration d'un texte ne fonctionne pas selon le modèle des vases communicants, où tout le contenu sémantique du premier médium serait déversé dans le second, mais selon celui d'une réappropriation subjective. Examinons de ce point de vue l'interprétation de Walter Crane.

Le fameux motif de la clef interdite est représenté de manière similaire en planches I et 4, à savoir dans les mains de Barbe bleue, qui présente les clefs à sa femme d'abord pour lui expliquer leur usage, ensuite pour lui faire comprendre son courroux. Dans les deux cas, le trousseau est présenté au niveau de la taille de Barbe bleue, ce qui pourrait laisser entendre que

27. B. Bettelheim, Psychanalyse des contes de fées, trad. fr. T. Carlier, Paris, Presses Pocket, 1976, p. 439-445. Les analyses de Bettelheim confinent parfois à l'absurde, comme quand il suppose que la femme de Barbe bleue et ses amies ont eu des relations sexuelles pendant l'absence du mari (p. 442), interprétation que rien dans le texte de Perrault ne permet de conforter. 
l'illustrateur aurait ainsi exprimé, de manière plus ou moins consciente, l'idée d'une parenté entre clefs et organe sexuel masculin. D'autant plus qu'en planche i la jeune épouse empoigne le trousseau de clefs à l'endroit tout à fait signifiant de l'entrejambe de son mari. Toutefois, les indices s'arrêtent là. En effet, en planche I, ce sont les autres clefs, celles du gardemeubles, des coffres et cassettes à trésors qui sont empoignées par la femme, et non la clef du cabinet interdit, qui est tenue horizontalement par Barbe bleue, justement de manière à la détacher visuellement des autres. Même chose en planche 4 , où la clef ensanglantée est tenue à part du reste du trousseau. La position du trousseau au niveau de la taille, dans les deux planches, peut par ailleurs s'expliquer autrement : c'est une manière de donner au motif, à des fins symboliques tout autant que narratives, une importance visuelle en le plaçant à chaque fois au niveau de l'axe horizontal médian, voire, en planche 4, en le plaçant au fil d'une ligne oblique construite d'un côté par le bras droit de Barbe bleue et de l'autre par le corps de sa femme agenouillée et les plis de sa robe. Dans cette dernière planche, le trousseau se trouve d'ailleurs non seulement le long de l'axe horizontal médian, mais aussi de l'axe vertical médian : c'est véritablement le centre de la composition, au milieu de la double page, qui divise spatialement et symboliquement Barbe bleue et sa femme. Rien d'étonnant du coup à ce que le symbole soit à mi-corps : il en va de la composition narrative de l'image. Toutefois, la manière dont, en planche I, le trousseau est empoigné par la mariée le désigne comme objet du désir : non encore la clef du cabinet interdit, mais les clefs des trésors de Barbe bleue. Le désir est clairement ici celui du lucre plutôt que de la luxure, et il en va des clefs ici non comme d'un phallus désigné comme tel, mais, pour reprendre Bellemin-Noël, comme d'un «machin [qui] représente par une image prégnante ou par un mot plus ou moins sacralisé tout ce qui est susceptible d'établir un lien, de concrétiser une appropriation, de véhiculer une quête de plaisir, voire de jouissance ${ }^{28}{ }^{\prime}$. Ce qui nous amène à une interprétation non sexuelle, mais politique, qui demande de revenir aux armoiries de Barbe bleue, sur lesquelles Walter Crane insiste à plusieurs reprises.

28. J. Bellemin-Noël, Les Contes et leurs fantasmes, Paris, Presses universitaires de France, I983, p. 40. 


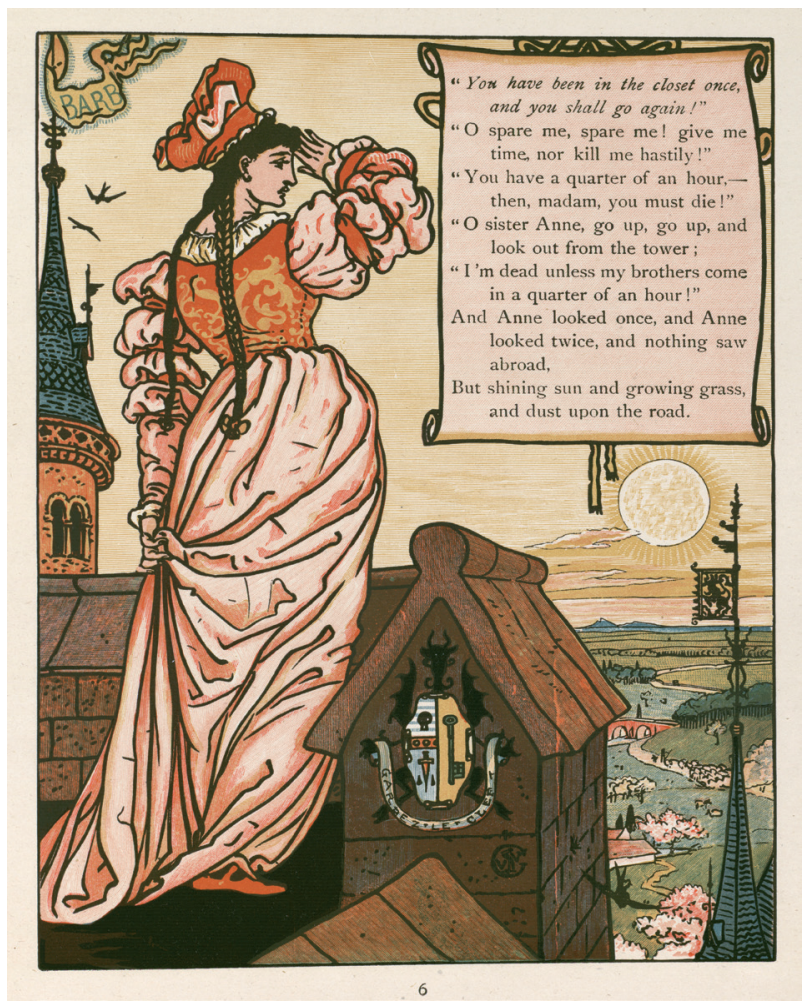

Fig. 5. - Walter Crane, chromoxylographie d'Edmund Evans, planche 5 (p. 6) de Bluebeard, Londres, John Lane, 1898.

Université François-Rabelais, Tours.

Ces armes imaginaires peuvent se blasonner ainsi : «Parti, au un d'azur à la fasce de gueules à trois besants d'or, au crâne de sable en chef et à un poignard d'or accompagné de deux gouttes de sang au naturel en pointe, au deux d'or à la clef d'azur ${ }^{29}$. On a déjà vu que la présence du poignard et de la tête de mort (ou en planche $6 \mathrm{du}$ pendu) inscrivait Barbe bleue sous le signe de la violence et de la mort. Or, le bandeau rouge à trois cercles jaunes («fasce de gueules à trois besants d'or») a aussi une importance qui n'est pas que décorative : le motif appelle une interprétation qui est celle de richesses mal acquises, dans le sang et la violence, et désigne

29. Nous remercions Jérome de Sousa Pinto pour nous avoir aidé à blasonner et interpréter ces armoiries. Celles-ci différant d'une planche à l'autre, ce blason est celui de la planche 5 . 
ainsi les crimes passés de Barbe bleue ${ }^{30}$. Bluebeard, selon Walter Crane, est donc surtout l'histoire d'un bien acquis par la violence, et la clef est une clef aux trésors, comme le signale suffisamment le fond "d'or" (jaune) sur lequel est inscrit le motif.

Le fait est par ailleurs que Walter Crane fait de Barbe bleue un noble, alors que le texte d'origine de Perrault (et la traduction de Planché) laissait clairement entendre que Barbe bleue est un roturier, notamment à travers l'évocation de son arme, un "coutelas» («cutlass» chez Planché) qui n'est pas une épée, mais qui en devient pourtant une dans l'illustration de l'artiste anglais (fig. 6). Autre détail, le château, qui n'est pas l'ensemble de "maisons» évoqué par l'académicien ( "houses» chez Planché), et permet de fait une interprétation aristocratique du personnage ${ }^{31}$. On peut même préciser : une noblesse d'épée, qui doit sa richesse à un glaive ensanglanté plutôt qu'au commerce et aux investissements d'une noblesse de robe.

Troisième élément à prendre en compte, Walter Crane était, même à cette époque ${ }^{32}$, très intéressé par la cause politique du socialisme, et était donc sensible aux questions de lutte des classes. Au point que, pour Jack and the Beanstalk, on a vu que l'artiste se fait l'écho d'une réécriture socialiste du conte traditionnel anglais par Linton, où l'ogre peut légitimement être volé par Jack dans la mesure où il avait lui-même volé la classe laborieuse de laquelle Jack est issu. Aurait-on affaire à une telle réécriture dans Bluebeard, paru la même année? Assurément non, du moins pas de manière manifeste. Relisons toutefois l'excipit de l'album (fig. 7) en le comparant à celui de l'original de Perrault.

30. La fasce de gueules est remplacée en planche 6 par une fasce d'azur à cinq besants d'or, ce qui amoindrit l'idée d'une richesse construite dans un bain de sang, mais permet néanmoins de renvoyer à la couleur de la barbe du propriétaire des armoiries, le poignard et la figure de pendu suffisant à signifier dans ce cas l'idée d'une richesse mal acquise : la simple juxtaposition des motifs de la clef, du poignard, de la mort et des pièces d'or suffit à raconter l'histoire de Barbe bleue, sans même le symbolisme des couleurs qui varie d'une planche à l'autre. L'héraldique de W. Crane n'est ni rigoureuse ni uniformisée, mais reste symboliquement motivée.

31. Ce dernier indice n'est pas en lui-même concluant, dans la mesure où dès le manuscrit de I695 débute une interprétation visuelle de la demeure de Barbe bleue comme un château avec pont-levis. Toutefois, il converge avec les autres détails (épée, proximité avec les portraits aristocratiques de la Renaissance, et surtout armoiries) pour faire de manière marquée de ce personnage un aristocrate - ce qui n'était pas le cas dans les illustrations de i695 et I697 des contes, plus ambiguës, où malgré le château il n'y a pas d'armoiries, et où surtout Barbe bleue lève un sabre et non une épée.

32. L'engagement concret de l'artiste dans les différents partis et ligues socialistes date du début des années I880, mais dès les années I860 il est sensibilisé à la «cause» par son maître W. J. Linton. 


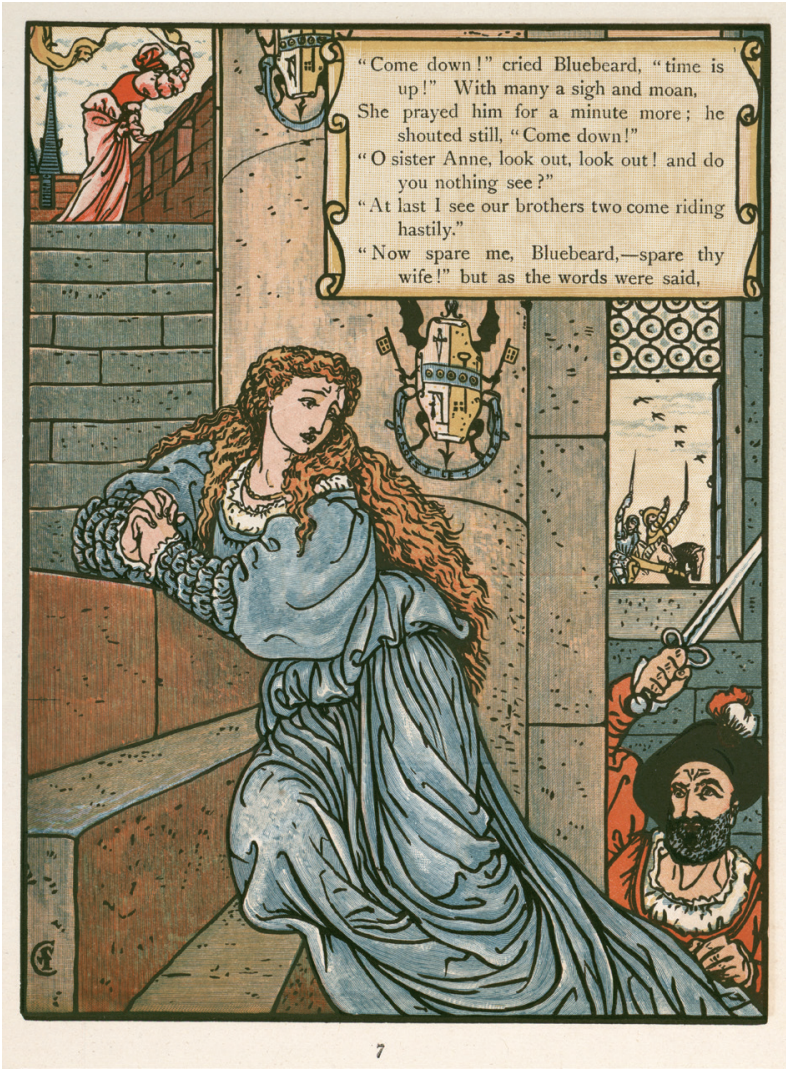

Fig. 6. - Walter Crane, chromoxylographie d'Edmund Evans, planche 6 (p. 7 ) de Bluebeard, Londres, John Lane, 1898.

Université François-Rabelais, Tours.

In rushed the brothers with their swords, - they cut the murderer down, And saved their sister's life, and gained much glory and renown; And then they all with gold and plate and jewels rare made free, And ever after lived content on Bluebeard's property ${ }^{33}$.

33. «Et au moment où le cruel glaive de Barbe bleue descendait sur sa tête, / S'engouffrèrent les deux frères avec leurs épées, - ils abattirent le meurtrier, / Et sauvèrent la vie de leur sœur, et gagnèrent bien de la gloire et du renom; / Et alors tous, avec de l'or, des trésors et joyaux rares, furent libérés, / Et ils vécurent heureux sur la propriété de Barbe bleue.» 
Et le texte de Perrault :

Ils lui passèrent leur épée au travers du corps, et le laissèrent mort. [...] Il se trouva que la Barbe bleue n'avait point d'héritiers, et qu'ainsi sa femme demeura maitresse de tous ses biens. Elle en employa une partie à marier sa sœur Anne avec un jeune Gentilhomme, dont elle était aimée depuis longtemps; une autre partie à acheter des Charges de Capitaine à ses deux frères; et le reste à se marier elle-même à un fort honnête homme, qui lui fit oublier le mauvais temps qu'elle avait passé avec la Barbe bleue.

On voit que dans les deux cas la fratrie profite des richesses laissées par le défunt. Mais la dimension matrimoniale et amoureuse décrite par Perrault, où la veuve comme sa sœur Anne profitent de leur fortune pour faire de bons mariages qui correspondent à leurs sentiments, est totalement éclipsée par Lucy et Walter Crane. De même pour la légitimation juridique de l'héritage (c'est parce que Barbe bleue n'a pas d'enfants que sa veuve peut en bénéficier) : dans le texte du toy book, aucune précaution de ce genre; dans le mouvement même de la mort de Barbe bleue, sa fortune change de mains. Autant qu'une vengeance suite aux brutalités du seigneur vêtu de rouge, on voit donc s'esquisser en filigrane un « retour de fortune", où une famille désargentée peut récupérer les trésors mal acquis d'un aristocrate criminel. Le fait même que le conte se termine sur cette notion de "propriété " permet d'insister sur cet aspect socio-économique : les opprimés, plus qu'hériter de la fortune de Barbe bleue, se l'approprient purement et simplement.

Un dernier élément, enfin : le coffre à trésors comme boîte de Pandore. Morna O’Neill a longuement commenté les significations de ce motif de la boîte de Pandore dans l'œuvre de Crane, et nous n'y reviendrons pas en détail ${ }^{34}$, mais on peut rappeler que pour l'artiste le motif est le lieu d'une interrogation sur les maux de la société et sur l'espoir de l'avènement d'une société meilleure - c'est-à-dire d'une société socialiste. Partant, la profanation du cabinet secret de Barbe bleue a le mérite de mettre en branle l'ordre social existant, de secouer les non-dits du capital en bravant les interdits, et de permettre ainsi une libération finale.

On est cependant loin ici de la révolution du prolétariat avec confiscation du capital de la bourgeoisie : il est ici affaire essentiellement de mariage, et non de travail, la femme reste une "lady» de bonne condition sociale, sinon économique, et les modifications de l'excipit ont sans doute

34. M. O'Neill, Walter Crane, The Arts and Crafts, Painting and Politics, I875-I890, New HavenLondres, Yale University Press, 20IO, p. II3-I37. 
une raison technique - il faut condenser la fin de l'histoire pour la faire entrer dans cinq vers - autant qu’idéologique.

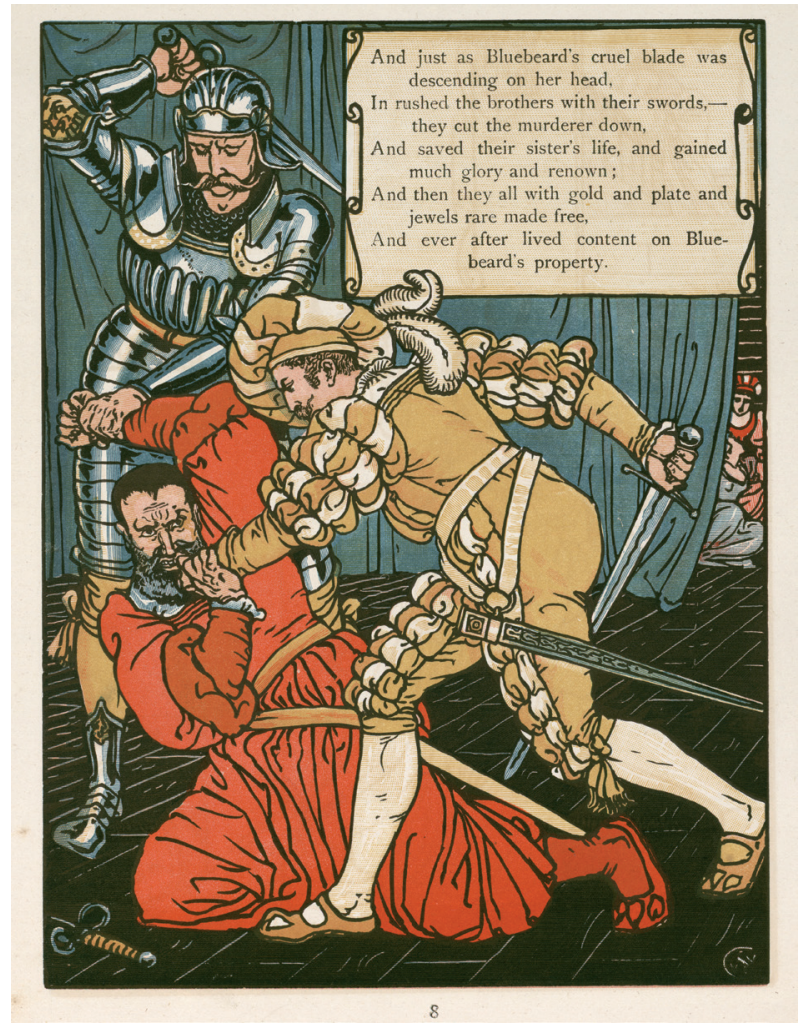

Fig. 7. - Fig. 7 : Walter Crane, chromoxylographie d'Edmund Evans, planche 7 (p. 8) de Bluebeard, Londres, John Lane, 1898. Université François-Rabelais, Tours.

Il n'empêche que les modifications textuelles convergent avec d'autres éléments visuels (la symbolique des armoiries, la boîte de Pandore, la représentation de Barbe bleue en aristocrate) vers une idée "clef» : Barbe bleue est un noble ayant acquis ses richesses dans la violence, et cette richesse lui est reprise dans la violence. On peut donc interpréter la clef comme celle du secret bien gardé du capital, à savoir que les richesses des classes dominantes ont été acquises dans le sang des classes opprimées. Tel est - aussi - le sens du récit de Perrault si on le débarrasse, comme l'ont fait Lucy et Walter Crane, de sa dimension galante, et qu'on le synthétise en textes et en images dans un sens bien particulier. Que cette dimension politique du conte ne soit énoncée dans l'image et le texte que de manière 
latente et non de manière manifeste, on le doit au tact de l'illustrateur, qui se fait un devoir de ne rien ajouter au texte d'origine qui n'y soit contenu «en puissance». Contrairement à George Cruikshank qui avait, une vingtaine d'années plus tôt, réécrit des contes de fées classiques pour en faire des pamphlets anti-alcoolisme, Walter Crane sait rester discret, et interprète le texte plus qu'il ne l'adapte aux problématiques qui sont les siennes.

\section{Conclusion}

Plutôt qu'une interprétation directement sexuelle du conte, donc, Walter Crane ouvre à une interprétation matrimoniale, qui considère la relation amoureuse dans sa dimension simplement «légale»: sociale et patrimoniale. Le désir de la mariée est initialement attisé par le train de vie de son mari ("But as he was so rich and grand, and led a merry life, I A lady he contrived at last to induce to be his wife." — "Mais comme il était très riche et majestueux, et menait une vie joyeuse, / Il réussit enfin à persuader une dame de devenir son épouse.»), et c'est seulement dans un second temps que la curiosité l'amène à vouloir en savoir plus. La tentation n'est sans doute plus alors - mais comment s'en assurer? même le texte de Perrault ne nous le dit pas clairement - celle du lucre, mais celle, plus sourde et moins évidente à circonscrire, de l'interdit. En somme, le désir non plus de posséder mais de savoir - soit en l'espèce de voir ce qui ne peut être représenté (planche 3). Le secret sanglant de la fortune de Barbe bleue dévoilé, la course contre la montre de la délivrance est enclenchée : comme l'espoir était resté au fond de la boîte de Pandore au milieu des maux répandus, il subsiste encore sur le coffre de mariage sous la forme d'un sablier (planche 4) - il reste du temps, ne cédons pas au désespoir.

On pourra sans doute juger que cette "désincarnation» de la relation amoureuse latente dans le conte de Perrault a pour cause un certain refoulement à l'œuvre chez l'artiste victorien. Il reste que son interprétation a l'intérêt de faire jaillir une partie du sens latent du conte à travers un langage graphique propre, associant symbole et ornement ${ }^{35}$. Et l'originalité de s'attaquer, certes de manière couverte, au problème de l'origine

35. Sur la dimension sémantique — allégorique — de l'ornement chez Crane, voir ibid., p. I49I52, mais aussi G. Smith, «Developing a public language of art», dans G. Smith et S. Hyde (éd.), ouvr. cité, p. I3-23, et W. Crane lui-même, "Of ornament and its meaning", dans Ideals in Art, Londres, G. Bell and Sons, I905, p. I02-I09 : "I still think [...] that an ultimate symbolical meaning, however veiled, gives an interest and a dignity to any piece of ornament, as well as a certain vitality which it could not otherwise possess." ("Je pense malgré tout [...] qu'une signification symbolique 
politique de la fortune de Barbe bleue. Les enfants ont-ils été réceptifs à un tel étalage d'allusions savantes et symboliques? Il est manifeste que les toy books de Walter Crane sont à cette époque au moins autant présents dans les bibliothèques des esthètes fin de siècle que dans les nurseries de la bourgeoisie victorienne ${ }^{36}$ : ils invitent de fait à une double lecture, qui se déploie selon le degré d'intérêt, d'attention et de culture du lectorat. Peut-être est-ce là aussi, à l'instar des contes de Perrault, une raison de leur succès.

ultime, quelque voilée qu'elle soit, offre un intérêt et une dignité à n'importe quel ornement, ainsi qu'une certaine vitalité qu'il ne pourrait sinon posséder.» — Nous soulignons.)

36. A. Lundin, Victorian Horizons, The Reception of the Picture Books of Walter Crane, Randolph Caldecott and Kate Greenaway, Lanham-Londres, Children's Literature Association, Scarecrow Press, 200I, notamment p. 29-3I et 35-37. 